\title{
A NOVEL BRST APPROACH IN GENERALIZING THE JACKIW-NAIR ANYON
}

\author{
Subir Ghosh \\ Physics Department, Gobardanga Hindu College \\ 24 Pgs. (North), West Bengal, India \\ S. Mukhopadhyay \\ Saha Institute of Nuclear Physics \\ 1/AF Bidhannagar, Calcutta 700064, India.
}

\begin{abstract}
A novel BRST quantization is described, which is applied in generalizing the JackiwNair construction of anyon. We have explicitly shown that the matter states connected to an unconventional ("non-zero") BRST ghost sector are physical. They are identified to the Jackiw-Nair system in a particular gauge. Also for the first time an indepth analysis of the present kind for a reducible constraint system, ( where the constraints are not independent), has been performed.
\end{abstract}


In this Letter, we have developed a relativistic Lagrangian model of anyon, which is a generalization of the Jackiw and Nair (JN) construction [1]. In a particular gauge our theory reduces to that of the $J N$ anyon. We have performed a BRST quantization of our [2] original spinning particle model of anyon, along the lines of Marnelius [3]. In the process we have introduced a novel idea of utilizing an unconventional BRST ghost sector. Indeed, this new scheme is applicable in a much wider class of physical models, although some formal details have to be worked out. Apart from these two major results, there are some more interesting aspects of a rather technical nature : we have carried through the quantization [3] in a qualitatively different and also much more involved type of system [2], which is known as a reducible constrained system [4], meaning that the constraints are not independent. This reducibility crucially affects the final results and has compelled us to use the more rigorous

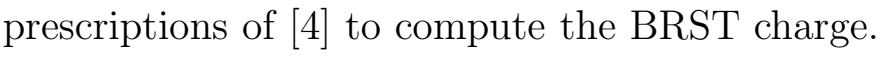

What is the motivation behind constructing new models of anyon? The conventional model [5] has been that of an interacting theory of a statistical gauge field (with Chern-Simmons dynamics) coupled with point particles (in quantum mechanics) or with bosonic or fermionic matter fields (in field theory). Elimination of the gauge field leaves the matter particle endowed with anyonic spin and statistics. However, the Chern-Simmons construction is non-relativistic and essentially classical. It has been argued [6] that residual effects remain at the quantum level even after removal of the gauge field. All these have motivated the search for minimal relativistic models of anyons where the complications due to an interacting theory are absent. Some of the successful models are [1, 7, 2].

Let us briefly recapitulate our model [2]. It is represented by a point particle, with a rotating frame attached to it moving along a world line. The instantaneous position and orientation of the particle with respect to a space fixed frame is denoted by a Poincare group element $\left(x^{\mu}(\tau), \Lambda^{\mu \nu}(\tau)\right)$, where $\tau$ is some parameter. Our metric is $g^{00}=-g^{11}=-g^{22}=1$. Linear and angular velocities are given by $u^{\mu}=\frac{d x^{\mu}}{d \tau}$ and $\sigma^{\mu \nu}=\left(\Lambda^{T} \frac{d \Lambda}{d \tau}\right)^{\mu \nu}$. The reparametrization invariant Lagrangian is

$$
L=\left(M^{2} u^{2}+\frac{1}{2} J^{2} \sigma^{2}+M J \epsilon^{\mu \nu \lambda} \sigma_{\mu \nu} u_{\lambda}\right)^{\frac{1}{2}},
$$

where $M, J$ are parameters having physical significance of mass and spin respectively. The canonically conjugate linear and angular momenta $P_{\mu}$ and $S_{\mu \nu}$ are related by the constraints which could be regrouped in the form

$$
\Psi_{1}=\epsilon_{\mu \nu \lambda} S^{\mu \nu} P^{\lambda}-2 M J \approx 0, \quad \Psi_{2}=P^{2}-M^{2} \approx 0, \quad \bar{\Psi}_{(0) \mu}=S_{\mu \nu} P^{\nu} \approx 0,
$$

of which the third one is a second class set. The Marnelius construction is best suited for First Class Constraints (FCC), which form a closed algebra. Hence we modify 
$\bar{\Psi}_{(0) \mu}$ by $\Psi_{(0) \mu}=S_{\mu \nu}\left(P^{\nu}+\Lambda^{0 \nu}\right)$ and the resulting set of FCC $\Psi_{A}, A=0,1, \mu$, satisfy the commutation relations,

$$
\begin{gathered}
{\left[\Psi_{(0) \mu}, \Psi_{(0) \nu}\right]=i S_{\mu \nu} \Psi_{2}-i\left(\Psi_{(0) \mu} P_{\nu}-\Psi_{(0) \nu} P_{\mu}\right),} \\
{\left[\Psi_{(0) \mu}, \Psi_{1}\right]=2 i \epsilon_{\mu \nu \lambda} P^{\nu} \Psi^{(0) \lambda}}
\end{gathered}
$$

A gauge fixing $P_{\mu}-\Lambda_{\mu}^{0}=0$ gives the original theory back. This set is not independent (namely reducible)because of the identity

$$
\Psi_{(0) \mu}\left(P^{\mu}+\Lambda^{0 \mu}\right)=0 .
$$

This feature of reducibility also agrees with the observation in [1].

We now follow the prescription of [10] for the BRST analysis. The constraints $\Psi_{A}$ are all bosonic and we introduce the auxiliary fields: bosonic Lagrangian multipliers $\left(\pi_{A}, \lambda^{A}\right)$ and fermionic ghost antighost pairs, $\left(\overline{\mathcal{P}}_{A}, \mathcal{C}^{A}\right)$ and $\left(\overline{\mathcal{C}}_{A}, \mathcal{P}^{A}\right)$. For the single reducibility condition fermionic multipliers $\left(\pi^{\prime}, \lambda^{\prime}\right)$ and the ghost antighost pairs and $\left(\overline{\mathcal{P}}^{\prime}, \mathcal{C}^{\prime}\right)$ and $\left(\overline{\mathcal{C}}^{\prime}, \mathcal{P}^{\prime}\right)$ a further set of extraghosts $\left(\pi^{\prime \prime}, \lambda^{\prime \prime}\right)$ and $\left(\overline{\mathcal{C}}^{\prime \prime}, \mathcal{P}^{\prime \prime}\right)$ are needed. A generic canonical pair is written as $\left(p_{A}, q^{A}\right)$ which satisfy the commutation relation $\left\{q^{A}, p_{B}\right\}=\delta_{B}^{A}$ for fermionic and $\left[q^{A}, p_{B}\right]=i \delta_{B}^{A}$ for bosonic degrees of freedom. Construction of the the quantum BRST charge, although straightforward, is quite involved (the system being reducible). Omitting the details we have

$$
\begin{aligned}
Q & =\Psi_{1} \mathcal{C}^{1}+\Psi_{2} \mathcal{C}^{2}+\Psi_{(0) \mu} \mathcal{C}^{(0) \mu}-\frac{i}{2} S_{\mu \nu} \mathcal{C}^{(0) \mu} \mathcal{C}^{(0) \nu} \overline{\mathcal{P}}_{2} \\
& -2 i P_{\mu} \epsilon_{\mu \beta}{ }^{\alpha} \overline{\mathcal{P}}_{(0) \alpha} \mathcal{C}_{(0)}^{\beta} \mathcal{C}^{1}+i P_{\mu} \overline{\mathcal{P}}_{(0) \nu} \mathcal{C}_{(0)}^{\nu} \mathcal{C}_{(0)}^{\mu} \\
& +\left(P_{\mu}-M \Lambda^{0}{ }_{\mu}\right) \mathcal{C}^{\prime} \overline{\mathcal{P}}^{\prime} \mathcal{C}_{(0)}^{\mu}+i\left(P^{\mu}+M \Lambda^{0 \mu}\right) \overline{\mathcal{P}}_{(0) \mu} \mathcal{C}^{\prime} \\
& +\frac{1}{2} \overline{\mathcal{P}}_{2}\left(\overline{\mathcal{P}}_{(0) \mu} \mathcal{C}_{(0)}^{\mu}-i \mathcal{C}^{\prime} \overline{\mathcal{P}}^{\prime}\right)
\end{aligned}
$$

This is same as that for the massive spinning particle in $(3+1)$ dimension [9] except for the first term which is due to the extra constraint. One can check the identity $Q^{2}=0$. The physical state must satisfy $Q \mid$ phys $>=0$.

The other crucial operator, the ghost number $N_{g}$ is

$$
N_{g}=\frac{1}{2}\left(\mathcal{C}^{A} \overline{\mathcal{P}}_{A}-\overline{\mathcal{P}}_{A} \mathcal{C}^{A}\right)-i\left(\mathcal{C}^{\prime} \overline{\mathcal{P}}^{\prime}-\overline{\mathcal{P}}^{\prime} \mathcal{C}^{\prime}\right)
$$

The operator ordering ambiguity is removed by following the prescription of [3]. This assigns the ghost number of any operator by $\left[N_{g}, \mathcal{O}\right]=n_{g} \mathcal{O}$, where $n_{g}$ is the ghost number. 
Now we start building the Hilbert space, concentrating first on the ghost sector. The vacua and the states are lebelled as $\mathcal{O} \mid>_{\mathcal{O}}=0$ and $\mid n_{g}>$. The fermionic sector reads

$$
\left.\left|-(+) \frac{1}{2}>_{i}=\right| 0>_{\overline{\mathcal{P}}_{i}\left(\mathcal{C}^{i}\right.}\right) \sim \overline{\mathcal{P}}_{i}\left(\mathcal{C}^{i}\left|0>_{\mathcal{C}^{i}\left(\overline{\mathcal{P}}_{i}\right)}, \quad{ }_{i}<m\right| n>_{j}=\delta_{i j} \delta_{m+n}, \quad i, j=0,1\right.
$$

For the other set $\left(\overline{\mathcal{P}}_{(0) \mu}, \mathcal{C}_{(0)}^{\mu}\right)$ we have

$$
\begin{gathered}
\left|-\frac{3}{2}>=\right| 0>_{\overline{\mathcal{P}}_{(0) \mu}},\left|-\frac{1}{2}>^{\mu}=\mathcal{C}_{(0)}^{\mu}\right| 0>_{\overline{\mathcal{P}}_{(0) \mu}}, \\
\left|+\frac{1}{2}>_{\mu}=\frac{1}{2} \epsilon_{\mu \nu \lambda} \mathcal{C}_{(0)}^{\nu} \mathcal{C}_{(0)}^{\lambda}\right| 0>_{\overline{\mathcal{P}}_{(0) \mu}}, \\
\left|+\frac{3}{2}>_{\mu}=\frac{1}{6} \epsilon_{\mu \nu \lambda} \mathcal{C}_{(0)}^{\mu} \mathcal{C}_{(0)}^{\nu} \mathcal{C}_{(0)}^{\lambda}\right| 0>_{\overline{\mathcal{P}}_{(0) \mu}}, \\
{ }_{\nu}<m \mid n>^{\mu}=\delta_{\nu}^{\mu} \delta_{m+n} .
\end{gathered}
$$

We can interchange $\mathcal{C}$ and $\mathcal{P}$ and that will flip the sign of the ghost number. The states corresponding to other ghosts could be constructed in this way.

However the bosonic sector consisting of ghosts, (as well as matter states), is infinite, unlike the fermionic one, since one can operate any number of bosonic creation operators on the vacuum. Thus there are a number of possible ghost states, which is not correct since there can not be physical ghost excitations [11. A natural way of removing this degenaracy is to restrict the space to states of finite norm only [3]. Since inner products such as $\left\langle p^{\prime} \mid p\right\rangle=\delta\left(p^{\prime}-p\right)$ are singular, a non-hermition state space is forced on us, where bras and kets are not hermition conjugate of each other. For its relations with the conventional Hilbert space and other details see references [10]. Consider the bosonic ghost to start with. Thus there are two possible 'ground states' $\mid 0>_{\overline{\mathcal{P}}^{\prime}}\left(\mid 0>_{\mathcal{C}^{\prime}}\right)$ which are annihilated by $\overline{\mathcal{P}}^{\prime}\left(\mathcal{C}^{\prime}\right)$ and a pair of towers of states can be built by operating $\mathcal{C}^{\prime}\left(\overline{\mathcal{P}}^{\prime}\right)$ repeatedly on them. So the Hilbert space consists of the following states.

$$
\begin{gathered}
\left|n>=\frac{\left(\mathcal{C}^{\prime}\right)^{n-1}}{\sqrt{(n-1) !}}\right| 1>_{\mathcal{P}^{\prime}}, \quad \mathcal{P}^{\prime}<0 \mid 0>_{\mathcal{P}^{\prime}}=1, \\
\left|-n>=\frac{\left(\mathcal{P}^{\prime}\right)^{n-1}}{\sqrt{(n-1) !}}\right|-1>_{\mathcal{C}^{\prime}}, \quad \mathcal{C}^{\prime}<0 \mid 0>_{\mathcal{C}^{\prime}}=1 .
\end{gathered}
$$

The construction of bosonic matter Hilbert space follows in a similar way. One has different choices for bra and ket states. The generic ket states are given by operating 
an arbitrary function of the position operator on the momentum 'ground state' (i.e. $\mid 0>_{P}$ with $\left.P_{\mu} \mid 0>_{P}=0\right)$ given by $\left|\Phi_{\alpha \beta \ldots}>=\Phi_{\alpha \beta \ldots}(x)\right| 0>_{p}$ where the bra states are given by $<x^{\prime} \mid C_{\alpha \beta \ldots}$ where $C_{\alpha \beta \ldots}$ is a covariant constant. For the spin sector one should use any conventional representation [12] and for that sector bra and ket states are hermitian conjugates of each other. Such a description of the matter Hilbert space is termed as the wave function (Schrodinger) representastion [3, 13].

Finally we have reached the most interesting part of our work: finding out the physical matter sector. Firstly we assumed that $\mid p h y s>$ is a direct product of the form $\mid$ matter $>\mid$ ghost $>$. Secondly the ghost sector consists of a sum of eigenspaces of $N_{G}$ with real ghost numbers. Hence $\mid$ phys $>$ is expanded in powers of ghosts [13,

$$
\mid \text { phys }>=|M>+| M>_{i}\left|G>_{i}+\right| M>_{i j} \mid G>_{i j}+\cdots
$$

where the number of indices in $\mid G>$ indicates the number of ghost operators on vacuum. The states $\mid M>_{i j \ldots}$ belong to the Dirac Hilbert space [8]. Normally the condition $Q \mid$ phys $>=0$ picks up the $\mid M>$ connected to zero ghost sector $\mid G>$ (namely $N_{g} \mid G>=0$ ) in case of Fock representation or the extreme ( \pm ) ghost number sector (namely $N_{g}\left|G>= \pm \frac{m}{2}\right| G>, m=$ number of constraints) in the wave function representation, which is relevant here. However the following comment in [13] is worth quoting :"The existence of non-trivial BRST cohomology at ghost number $\neq \pm \frac{m}{2}$ depends on the detailed structure of the theory. It will not concern us here because it does not appear to be related to the states of the Dirac method without ghosts." We want to emphasize, (as we will explicitly show), that in the present case the $\mid M>$ connected to a $\mid G>$ whose ghost number is not $\pm \frac{m}{2}$ is related to the physical states without ghost and this matter sector satisfies a generalized set of JN equations [回]. In fact this is forced on us as the matter part with conventional ghost sector turns out to be trivial, once $Q$ is operated on it. The cure for "non-zero" ghost number is also given in 13. One should suitably include the Lagrange multipliers and their conjugate momenta and extraghosts, which will change $Q$ and $N_{g}$ to

$$
\bar{Q}=Q+\pi_{A} \mathcal{P}^{A}+\pi^{\prime} \mathcal{P}^{\prime}+\pi^{\prime \prime} \mathcal{P}^{\prime \prime}, \quad \bar{N}_{g}=N_{g}-\frac{i}{2}\left(\lambda^{\prime} \pi^{\prime}+\pi^{\prime} \lambda^{\prime}\right)
$$

In this still enlarged space the ghost number of the physical sector just obtained is again zero. For the construction of the field equations we choose a sub-space given by

$$
\mid \text { phys }>=\left|-\frac{1}{2}>_{1}\right|-\frac{1}{2}>_{2}\left|-\frac{1}{2}>^{\alpha}\right| M_{\alpha}>\text {, }
$$

and its dual subspace which should involve the ghost states with opposite ghost number is

$$
<\text { phys }\left|=<M_{\alpha}^{\prime}\right|^{\alpha}<\left.\frac{1}{2}\right|_{1}<\left.\frac{1}{2}\right|_{2}<\frac{1}{2} \mid,
$$


where $<\left.M\right|^{\alpha}$ and $\mid M^{\prime}>_{\alpha}$ are the matter states represented in the wave function sector. Note that in $\mid$ phys $>$ we have retained the relevant variables only. Imposing the physicality condition $Q \mid$ phys $>=0$ and $<$ phys $\mid Q=0$ one gets the following restrictions on the matter space.

$$
\begin{aligned}
\left(\Psi_{1} \delta_{\alpha}^{\beta}-2 i \epsilon_{\alpha}^{\lambda} P_{\lambda} \mid M_{\beta}>\right. & =0, \quad \Psi_{2} \mid M_{\mu}>=0 \\
\epsilon^{\mu \nu \lambda}\left(\Psi_{\nu}+i P_{\nu}\right) \mid M_{\lambda}> & =0, \quad\left(P_{\mu}+\Lambda_{\mu}^{0}\right) \mid M^{\mu}>=0 \\
<M^{\prime \mu} \mid\left(\Psi_{\mu}+2 i P_{\mu}\right) & =0 .
\end{aligned}
$$

It is rather inconvenient to have both the bra states and the ket states restricted. We would like to have the bra states to be unrestricted. So we write the bra state as a projection on an unrestricted state by the projection operator $\Pi_{\alpha}^{\beta}=\left(\delta_{\alpha}^{\beta}-V_{\alpha} V^{-2} V^{\beta}\right)$ where $V_{\alpha}=\Psi_{\alpha}+2 i P_{\alpha}$ and we have written $V^{-2}$ formally. Since to get the field equations only the inner products are relevant one can replace $<M^{\prime}{ }_{\alpha} \mid$ and $\mid M_{\alpha}>$ by $<\tilde{M}_{\alpha}|,| \tilde{M}_{\alpha}>$ where $<M_{\alpha}\left|=<\tilde{M}^{\beta}\right| \Pi_{\alpha}^{\beta}$ and $\left|\tilde{M}_{\alpha}>=\Pi_{\alpha}^{\beta}\right| M_{\beta}>$. So the bra state remains unrestricted but the ket state has to satisfy the additional requirement

$$
\left(\Psi_{\mu}+2 i P_{\mu}\right) \mid M^{\mu}>=0
$$

We choose the matter sector states as $\left|M_{\alpha}>=\Sigma_{n} f_{\alpha, n}(x)\right| 0>_{p} \mid n>$ for ket state and $<x^{\prime}\left|<n^{\prime}\right|=<M^{\prime}{ }_{\alpha} \mid$ where $\mid n>$ is the representation of the spin sector. Taking the inner product of the equations $(19,21)$ with these ket states we have the following equations

$$
\begin{aligned}
\Psi_{2} f_{\mu, n}(x) & =0, \quad\left(\left(\Psi_{1}\right)_{n n^{\prime}} \delta_{\alpha}^{\beta}-2 i P_{\mu} \epsilon_{\alpha}^{\mu}{ }_{\alpha} \delta_{n n^{\prime}}\right) f_{\beta, n^{\prime}}(x)=0, \\
\left(\left(\Psi_{\mu}\right)_{n n^{\prime}}+2 i P_{\mu} \delta_{n n^{\prime}}\right) f_{n^{\prime}}^{\mu}(x) & =0, \quad \epsilon^{\mu \nu \lambda}\left(\left(\Psi_{\nu}\right)_{n n^{\prime}}+i P_{\nu} \delta_{n n^{\prime}}\right) f_{\lambda n^{\prime}}=0, \\
\left(P_{\mu} \delta_{n n^{\prime}}+M\left(\Lambda_{\mu}^{0}\right)_{n n^{\prime}}\right) F^{\mu}{ }_{, n^{\prime}}(x) & =0 .
\end{aligned}
$$

Here $P_{\mu}$ is written as an operator $-i \partial_{\mu}, J_{\mu}\left(S_{\mu \nu}=\epsilon_{\mu \nu \lambda} J^{\lambda}\right)$ and $\Lambda_{\mu}^{0}$ are written as their representations $<n^{\prime}\left|J_{\mu}\right| n>$ and $\left\langle n^{\prime}\left|\Lambda_{\mu}^{0}\right| n>\right.$. This is the generalized version of the field equation. The matrices representing $J_{\mu}$ serves the role of the 'gamma matrices' of the anyonic equation. This generalized set of equations (22) constitutes one of the main results of our work. Representation of the full $J_{\mu}, \Lambda_{\mu}^{0}$ system for the gauge symmetric version is still open. The problem of constructing a local field theoretic Lagrangian, the variation of which will reproduce the above set of equations is under study.

As promised earlier, we show how one can get the JN construction from our generalized version. As mentioned earlier we should take a gauge fixing. To get the JN construction from our equations the covariant gauge given by $\chi_{\mu}=P_{\mu}-M \Lambda_{\mu}^{0} \approx 0$ is prescribed. A generic gauge fixing requires introduction of extra terms in $Q$ which 
might affect the equations of motion. However this particular gauge is judicious in the sense that all the extra terms involve the auxiliary fields only and so do not modify the set of equations. After the gauge has been fixed we have to find a suitable representation in keeping with the gauge fixing. It should correspond to the arbitrary value of the spin. A suitable choice is given by the following [1, 12].

$$
\begin{aligned}
J^{0} \mid \lambda, n> & =(\lambda+n) \mid \lambda, n>, \\
J^{+} \mid \lambda, n> & =\sqrt{(2 \lambda+n)(n+1)} \mid \lambda, n+1>, \\
J^{-} \mid \lambda, n> & =\sqrt{(2 \lambda+n-1) n} \mid \lambda, n-1>,
\end{aligned}
$$

with $J^{ \pm}=J^{1} \mp i J^{2}, J^{-}\left|\lambda, 0>=0, J^{2}\right| \lambda, n>=\lambda(\lambda+1) \mid \lambda, n>$. This is the representation bounded from below and one can get the representation bounded from above by replacing $J$ by $-\tilde{J}$. Another point to note is that all the operators that can be obtained by changing the operator orderings in $\Psi_{(0) \mu}$ are reduced to the same expression under the covariant gauge fixing. So there is an ambiguity in the equation in the gauge fixed form. To resolve the ambiguity we are following the prescription that we first write down the equation in terms of the symmetrically ordered form

$$
\Psi_{\mu}^{(s)}=\frac{1}{2}\left[S_{\mu \nu}\left(P^{\nu}+M \Lambda^{0 \nu}\right)+\left(P^{\nu}+M \Lambda^{0 \nu}\right) S_{\mu \nu}\right],
$$

and then we fix the gauge. Now substitution of this representation will lead to the JN construction. We emphasize that in our analysis the mass shell condition, the Pauli Lubanski scalar equation, the subsidiary condition as well as the transversality condition of [1] all appear in an equal footing ( that is the latter are not invoked from the outside ).

Hence we conclude by noting that we have derived a set of generalized anyon wave equation which in a particular gauge is identical to the full JN construction. A novel BRST analysis which has led to the above construction explicitly demonstrates that the matter states connected to unconventional ghost sector can indeed be related to the Dirac matter sector without ghosts. In the process the Marnelius construction has been carried through in a reducible constraint system.

One of us (S. M.) would like to acknowledge the financial support of Council of Scientific and Industrial Research, India.

\section{References}

[1] R. Jackiw and V. P. Nair, Phys. Rev. D 43, 1933, (1991). 
[2] S. Ghosh, Phys. Lett. B 338, 235, (1994); S. Ghosh and S. Mukhopadhyay, Phys. Rev. D 51, 6843, (1995).

[3] R. Marnelius, Nucl. Phys. B 418, 353, (1994), and references therein.

[4] I. A. Batalin and E. S. Fradkin, Phys. Lett. B 122, 157, (1983); I. A. Batalin and G. Vilkovisky, Phys. Lett. B 69, 309, (1977).

[5] F. Wilczek and A. Zee, Phys. Rev. Lett. 51, 2250, (1983); C. Hagen, Ann. Phys. (N.Y.) 157, 342, (1984); see also F. Wilczek, Fractional Statistics and Anyon Superconductivity, World Scientific, Singapore, 1990.

[6] J. Hong, Y. Kim, and P. Y. Pac, Phys. Rev. Lett. 64, 2230, (1990); R. Jackiw and E. Weinberg, ibid, 2234 (1990).

[7] M. S. Plyuschay, Phys. Lett. B 262, 71, (1991); C. Chow, V. P. Nair and A. P. Polychronakos, Phys. Lett. B 304, 105, (1993).

[8] P. A. M. Dirac, Lectures in Quantum Mechanics, Yeshiva University, New York, Academic Press, 1967.

[9] N. K. Nielsen and Q. J. Quaade, Phys. Rev. D 52, 1204, (1995).

[10] R. Marnelius , Nucl. Phys. B 335, 395, (1990);

[11] R. Marnelius, Nucl. Phys. B 315, 638, (1989);

[12] H. J. Lipkin, Lie Groups for Pedestrians, North-Holland, 1965. B. Binegar, J. Math. Phys. 23, 1511, (1982).

[13] M. Henneaux and C. Teitelboim, Quntization of Gauge Systems, Princeton University press, Princeton, 1992. 